\title{
MICROPLÁSTICOS: USO NA INDÚSTRIA COSMÉTICA E IMPACTOS NO AMBIENTE AQUÁTICO
}

\author{
Julia Gabriela Matos Vargas ${ }^{\mathrm{a}}$, Vinicius Bueno da Silva ${ }^{\mathrm{a}}$, Lílian Karla de Oliveirab e Eduardo Ferreira Molina ${ }^{\mathrm{a}, *, \mathbb{B}}$ \\ ${ }^{a}$ Universidade de Franca, 14400-600 Franca - SP, Brasil \\ b'Instituto Federal de São Paulo, Campus Barretos, 14781-502 Barretos - SP, Brasil
}

Recebido em 04/10/2021; aceito em 04/01/2022; publicado na web em 07/03/2022

\begin{abstract}
MICROPLASTICS: APPLICATIONS IN THE COSMETIC INDUSTRY AND IMPACTS ON THE AQUATIC ENVIRONMENT. Environmental pollution from plastics and microplastics is currently one of the most relevant issues of the scientific community and cosmetic industry. In particular, microplastics are referred to as small pieces of plastic less than five millimeters $(<5 \mathrm{~mm})$ and do not dissolve in water, can be harmful to the ocean and aquatic life. Microplastics can be designated as i) primary - particles that are intentionally developed as micro sized plastic particles used in cosmetic formulations like microbeads, and ii) secondary microplastics - stem from all kinds of plastic material debris result of decomposition caused by UV radiation, mechanical stress (ocean waves) and wind action. This article gives an overview about plastics/microplastics, that are mainly used in personal care products and cosmetic. Moreover, we attempt to demonstrated some of the major problems that microplastics based materials can cause in the aquatic life leading to a human health concern. In this context, the interaction between microplastic-contaminants and possible effects in the environment or organisms was explore based on the literature analysis.
\end{abstract}

Keywords: polyethylene; microbeads; personal care products; organic contaminants; adsorption.

\section{INTRODUÇÃO}

Uma das obras literárias mais famosas do escritor Júlio Verne é conhecida como "Vinte Mil Léguas Submarinas". ${ }^{1}$ Essa obra de ficção científica é baseada em uma expedição marítima para encontrar um amedrontador monstro marinho. Esse monstro desconhecido foi criado em segredo pelo capitão Nemo, que através dos seus conhecimentos desenvolveu uma máquina a qual chamou de submarino. Desconhecido pela humanidade naquela época, este monstro começou a provocar acidentes com pequenas embarcações e navios, e passou a amedrontar o mundo. De acordo com a obra de Verne, "au fond de l'océan un objet long, fusiforme, parfois phosphorescent, plus vaste qu'une baleine suscite la curiosité des marins", ou seja, "no fundo do oceano um objeto comprido, fusiforme, às vezes fosforescente, maior que uma baleia desperta a curiosidade dos marinheiros". Assim, começava a caça a esse monstro. Mas qual a relação deste monstro que Verne relatou na sua obra com este trabalho? A resposta está associada ao problema atual dos ambientes aquáticos, em especial os oceanos devido à presença de "algo" não natural que afeta esses ambientes e provoca danos a organismos vivos.

Esse problema ambiental, no qual afeta todo um ecossistema marinho e organismos aquáticos vêm atraindo a atenção de pesquisadores e instituições ambientais na última década. Esse "monstro real", produzido em larga escala e lançado nos ambientes aquáticos é conhecido como microplástico (MPs). Os MPs vêm se tornado um campo emergente de estudo nas indústrias, universidades e centros de pesquisa. Assim, este artigo tem como foco apresentar questões ambientais sobre plásticos e microplásticos e as implicações dos cosméticos contendo esses materiais no ambiente.

Polímero segundo a definição da União Internacional de Química Pura e Aplicada (IUPAC) é um material que pode ou não conter outras substâncias que melhorem seu desempenho, como também reduza custos. ${ }^{2,3}$ Os polímeros se apresentam como uma imensa família de materiais versáteis e são amplamente usados pelas indústrias em

*e-mail: eduardo.molina@unifran.edu.br todo o mundo, por serem materiais de baixo custo, leves, moldáveis, duráveis e resistentes à corrosão, além de apresentarem propriedades de isolamento térmico e elétrico dependendo de sua composição e/ ou estrutura quimica. ${ }^{4}$

Os polímeros oferecem uma infinidade de recursos que permitem que a sociedade tenha acesso a alimentos seguros (embalagens), casas com eficiência energética, transporte verde, conectividade global, energias renováveis ou acessíveis, saúde higiênica, entre outros. Ainda hoje, a maioria dos materiais poliméricos são de origem fóssil, sendo produzidos a partir de derivados do petróleo, ou seja, a partir de recursos não renováveis. De acordo com a PlasticsEurope, ${ }^{5} \mathrm{em}$ termos de produção de plásticos no ano de 2019, países europeus produziram $16 \%$ de todos os plásticos usados no mundo, enquanto $17 \%$ foram produzidos nos EUA e 51\% na Ásia (Figura 1). Assim sendo, a produção de polímeros na Ásia provavelmente terá um impacto maior na poluição por plástico nos oceanos que nos demais países do mundo.

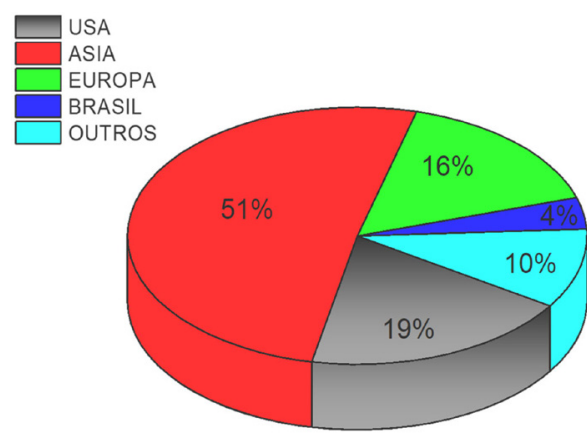

Figura 1. Produção de plásticos no mundo

O plástico é o tipo de resíduo mais comum encontrado nos oceanos. Segundo pesquisa feita pela organização não governamental World Wide Fund for Nature em 2019, ${ }^{6}$ o volume desse resíduo encontrado anualmente nos oceanos é de 10 milhões de toneladas. $\mathrm{O}$ Brasil é o $4^{\circ}$ maior produtor de resíduo plástico no mundo, produzindo em média 11 milhões de toneladas por ano. Os dois principais tipos de 
polímeros mais utilizados em nosso cotidiano são o polietileno (PE) e polipropileno (PP). Esses compostos são aplicados em diferentes áreas e podem ser encontrados em peças de automóveis, sacolas de compras, canos de água, dentre diversas outras funcionalidades. Para a obtenção desses materiais, é necessário uma reação de polimerização, na qual a reação se inicia com um constituinte primário denominado monômero. Um exemplo de reação de polimerização é apresentado na Figura 2, onde mostra de forma genérica a obtenção do PE. A presença de um catalisador geralmente sólido é exigida nestas reações de polimerização. ${ }^{7}$<smiles>C=C</smiles>

Etileno

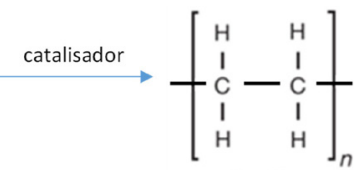

Polietileno
Figura 2. Exemplo de uma reação de polimerização para a conversão do monômero etileno para obtenção do polietileno com uso de catalisador

Polímeros à base de PE são os mais utilizados pelas empresas devido ao fácil manuseio, processabilidade e alta resistência química. Os produtos feitos com esses polímeros a base de PE são marcados com um símbolo de reciclagem circundando os números de " 1 " a " 6 ". A Tabela 1 mostra a nomenclatura, a aplicação com os respectivos símbolos de reciclagem dos polímeros à base de $\mathrm{PE}$, uma estimativa da massa $(\mathrm{em} \mathrm{kg}$ ) produzida anualmente e porcentagem reciclada dos polímeros ${ }^{8}$

Resíduos poliméricos podem possuir diferentes formas e tamanhos, porém aqueles encontrados em corpos aquáticos com menos de cinco milímetros $(<5 \mathrm{~mm})$ de comprimento são chamados de "microplásticos" (MPs). Estes materiais podem ser encontrados como fibras, esferas, fragmentos, pellets, entre outras formas. ${ }^{9-11}$
O termo "microplástico" foi relatado pela primeira vez em 2004 por Thompson e colaboradores ${ }^{12} \mathrm{e}$, desde então, tem sido amplamente encontrado em vários compartimentos ambientais e organismos vivos. ${ }^{13,14}$ Uma busca na base de dados "sciencedirect" $" 15$ utilizando a palavra "microplastic" mostra que na última década o número de pesquisas envolvendo esse tópico vêm crescendo de forma rápida e exponencial. Esse aumento no número de publicações pode ser observado na Figura 3, em que, no ano de 2011, foram publicados 87 artigos, e já no ano de 2020 foram publicados 2049 artigos referentes ao tema. No primeiro trimestre de 2021 foram publicados aproximadamente 1311 artigos sobre microplásticos, apresentando números maiores que todo o ano de 2019, possivelmente devendo ultrapassar o número de publicações do último ano (Figura 3).

As partículas de MPs podem ser classificadas como primárias quando são fabricadas originalmente para compor a formulação de determinados produtos, como esfoliantes, pasta de dental, medicamentos etc., e possuem comprimento e diâmetro estabelecidos durante seu desenvolvimento para serem utilizados em tais processos. ${ }^{16}$

MPs secundários são formados a partir da decomposição e fragmentação de plásticos maiores, geralmente devido ao desgaste ocorrido pela exposição dos fatores ambientais, por exemplo à ação das ondas, vento e radiação ultravioleta da luz solar. ${ }^{17}$ No ambiente, microesferas primárias e secundárias são difíceis de identificar e classificar, pois os MPs primários se comportam semelhantemente aos pequenos fragmentos oriundos da degradação. ${ }^{18}$

Um dos problemas no que diz respeito a biodiversidade é o fato de que esses tipos de polímeros (partículas, fibras, etc menores que 5 $\mathrm{mm}$ ) não podem ser removidos por processos utilizados nas estações de tratamento de águas residuais. Essas partículas passam por todo o processo de tratamento praticamente inalteradas, e assim chegam aos oceanos e demais corpos aquáticos. Os fragmentos dos plásticos e as substâncias retidas nestes materiais (via absorção/ adsorção) podem

Tabela 1. Principais polímeros produzidos à base de PE, nomenclaturas e respectivas fórmulas estruturais. Estimativa da massa produzida (kg) e porcentagem reciclada de PET, PEAD, PVC, PEBD, PP e PS ${ }^{8}$

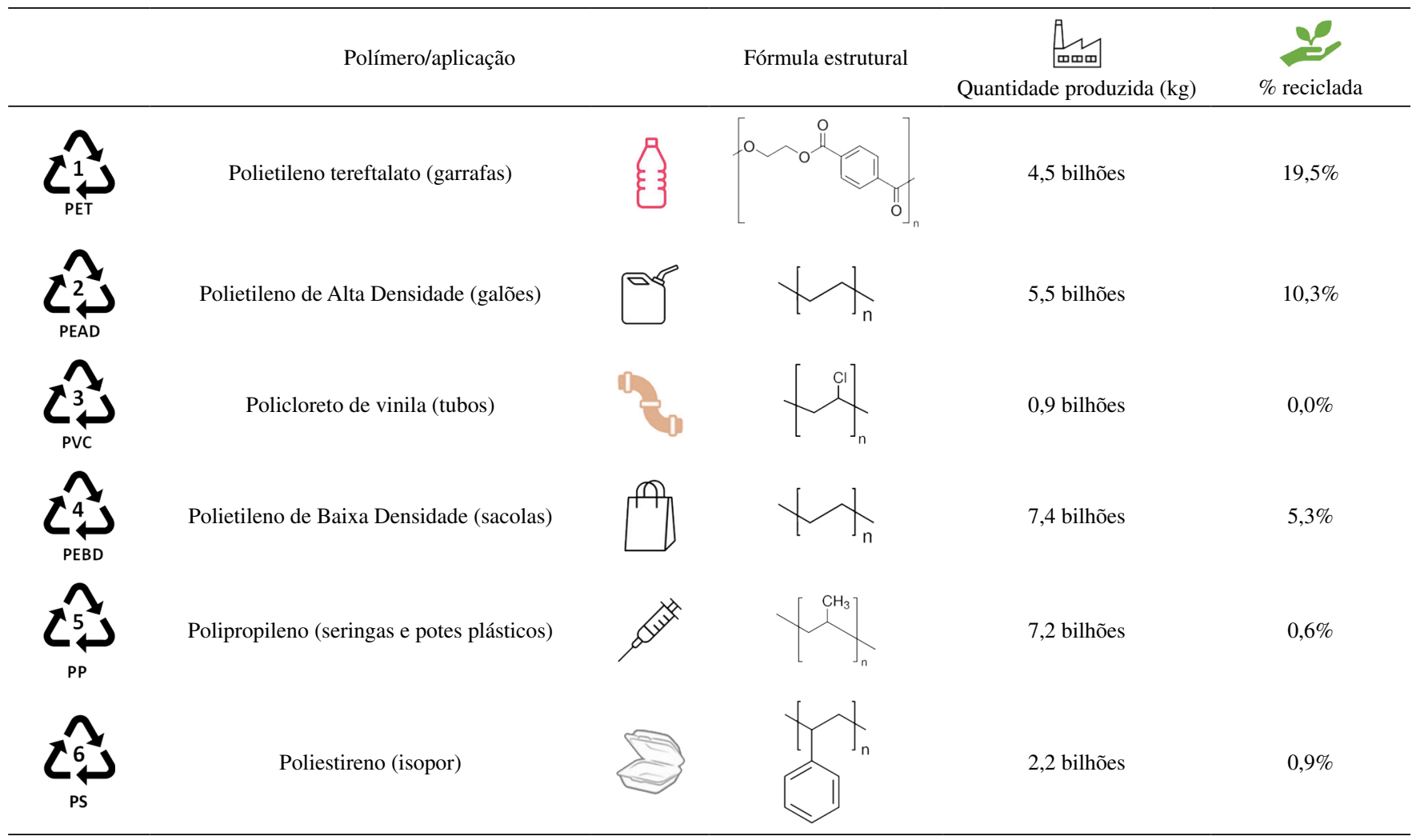




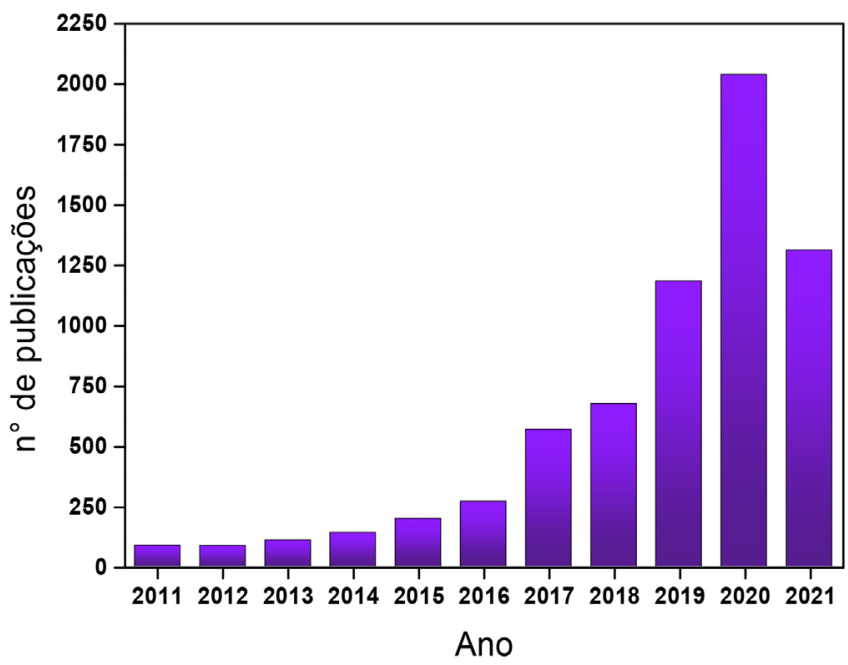

Figura 3. Número de publicações na última década sobre o tema microplásticos. Consulta utilizando a base de dados "sciencedirect" e palavra-chave "microplastic."15 O número de publicações do ano de 2021 refere-se aos dados do $1^{\circ}$ trimestre

ser ingeridos e acumuladas por organismos aquáticos, contaminando toda a cadeia biológica. ${ }^{19}$ Dessa forma podem desencadear efeitos catastróficos no ambiente, na biota marinha e alcançar níveis tróficos superiores.

Em síntese, é evidente que os plásticos/microplásticos são poluentes e produzidos em largas escalas na sociedade contemporânea, porém, seus impactos ecológicos ainda são investigados e debatidos pela comunidade cientifica. ${ }^{20,21}$ Desse modo, este trabalho tem o objetivo de reunir informações presentes na literatura acerca dos MPs, suas aplicações na indústria cosmética e os possíveis impactos nos ambientes aquáticos.

\section{Panorama sobre a utilização de microplástico em produtos de higiene pessoal e cosméticos}

Uma pesquisa de mercado realizada no de 2018 pela empresa Euromonitor Internacional, destaca o Brasil em $4^{\circ}$ lugar entre os países que mais consome cosméticos e produtos para cuidados pessoais do mundo, totalizando US\$ 30 bilhões de faturamento. No panorama mundial, entre as categorias mais consumidas estão a de higiene oral e produtos para a pele, representando a terceira e oitava colocação, respectivamente. Em ambas as categorias citadas se encontram produtos que contêm microesferas plásticas como produtos para higiene bucal e esfoliantes..$^{22,23}$

A esfoliação é um processo que detém a função de auxiliar na renovação celular da camada córnea da epiderme, retirando células superficiais repletas de queratina com baixo teor de hidratação e sem a vitalidade desejada. ${ }^{24}$ Tal procedimento é realizado com cosméticos de ação esfoliante que tem em sua composição partículas sólidas de uso tópico, de acordo com a Resolução da Diretoria Colegiada (RDC) $n^{\circ}$ 4 de 30 de janeiro de 2014 são classificados como grau 1 esfoliantes do tipo mecânico ou como de grau 2 esfoliantes do tipo químico. ${ }^{25}$

Para obter uma melhor ação de descamação das células superficiais do epitélio, microesferas são incorporadas a formulações de esfoliantes (grau 1) para obter a renovação celular através da fricção mecânica. ${ }^{26}$ Essas microesferas são partículas sólidas de plástico que são usadas intencionalmente como ingredientes de produtos; medem 5 milímetros ou menos e são insolúveis em água. É relatado que cerca de $93 \%$ das micropartículas utilizadas para a fabricação de cosméticos e produtos de cuidados pessoais são MPs, como polietileno (PE), polipropileno (PP), politeraftalato de etilieno (PET), polimetilmetacrilato (PMMA) e nylon. ${ }^{27,28}$
Não se restringindo apenas a esfoliantes, esferas de MPs também são encontradas em produtos de cuidados pessoais, como cremes dentais e shampoos..$^{29-31}$ Bila e colaboradores ${ }^{32}$ relataram que apesar de não conter informações no rótulo de um determinado produto utilizado na higiene bocal, verificaram a partir de análises laboratoriais um percentual de $0,7 \%(\mathrm{~m} / \mathrm{m})$ de MPs $(\mathrm{PE})$ no produto analisado. Após serem utilizadas na rotina de cuidados pessoais, estas microesferas plásticas vão para o tratamento de águas residuais das cidades, ${ }^{18}$ e geralmente percorrem todas as etapas de tratamento das estações permanecendo intactas. Portanto, esses materiais são aportados nos ambientes muitas vezes sem nenhum tratamento prévio. ${ }^{33}$ Alguns trabalhos relatam a presença de até $10 \mathrm{mg} / \mathrm{mL}$ de microesferas em esfoliantes facias ${ }^{31,34}$ e que uma pessoa utiliza em média $15 \mathrm{mg}$ de pequenos detritos plásticos por dia. ${ }^{30}$

A literatura sugere que um dos principais meios de poluição por materiais poliméricos de tamanho micro e nano em sistemas aquáticos são as pequenas esferas plásticas utilizadas em cosméticos, fragmentos esses que são descartados em águas residuais domésticas. ${ }^{35,36}$ As microesferas presente em esfoliantes, cremes dentais, loções e outros tipos de cosméticos são um poluente em potencial, pois quando utilizados seus resíduos são lançados diretamente para os esgotos domésticos, seguindo para estações de tratamento, rios, e finalmente alcançando o ambiente marinho. ${ }^{29}$ De acordo com Gouin e colaboradores, ${ }^{27}$ a emissão de microesferas originadas de produtos cosméticos representam entre $0,1 \%-1,5 \%$ (porcentagem em massa) da quantidade total de detritos plásticos lançados no mar do Norte. Apesar da baixa porcentagem, os valores apresentados são significativos já que estas partículas se encontram em pequenos tamanhos. No ano de 2015, foi relatado o uso de 4100 toneladas de microesferas em produtos cosméticos nos países da União Européia. ${ }^{27} \mathrm{O}$ uso de gel contendo microesferas durante um banho pode enviar cem mil (100.000) microesferas para rios e oceanos. ${ }^{37}$ A Figura 4 apresenta exemplos de MPs utilizados em produtos de higiene pessoal e cosméticos com irregulares superfícies e formas.

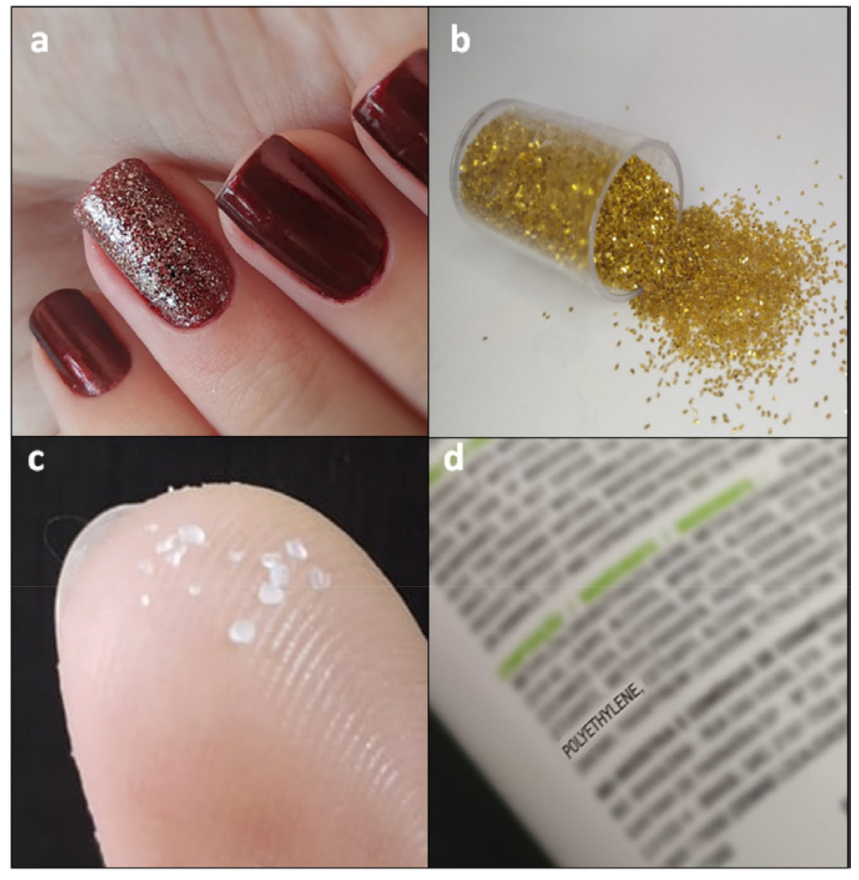

Figura 4. (a) MPs utilizados como efeito de arte para unhas, (b) glitter de plástico utilizados em cosméticos e como intuito para fins decorativos, (c) microesferas extraidas de produto cosmético e $(d)$ exemplo de um ingrediente microplástico listado em um produto cosmético 


\section{Comportamento de fragmentos plásticos em estações de tratamento}

Em geral, os polímeros possuem baixa densidade e são liberados nos rios e lagos, para posteriormente serem aportados nos oceanos. ${ }^{38}$ Há uma grande dificuldade em remover MPs por processos padrões de tratamento de águas; tais partículas poliméricas podem passar por estações de tratamento praticamente ilesas, ou seja, sem alterar suas propriedades físico-químicas..$^{29,39,40}$ Estudos mostram que os MPs são uma categoria de resíduos nocivos aos ambiente aquáticos, não só devido à sua grande capacidade de atravessar barreiras biológicas, mas também em virtude das implicações nos mecanismos de bioacumulação e biomagnificação de outros compostos poluentes. ${ }^{16}$

Um fator preocupante é que em países em desenvolvimento cerca de 80 a $90 \%$ das águas residuais não são tratadas, sendo aportadas em zonas costeiras. ${ }^{41}$ Mesmo quando o processo de tratamento de água é realizado, pode-se encontrar partículas poliméricas após o tratamento. ${ }^{42,43}$

\section{Implicações do microplásticos como poluente no ambiente}

A literatura reporta a capacidade dos plásticos em adsorver/ transportar substâncias tóxicas, persistentes e bioacumuláveis presentes em pequenas quantidades em ambientes aquáticos. Essa capacidade pode estar associada a (i) morfologia irregular, (ii) hidrofobicidade e (iii) elevada área superficial, onde os MPs podem potencializar a condição de vetores de poluentes persistentes/emergentes. ${ }^{19,44,45}$ Exemplos destes contaminantes são bifenilos policlorados, hidrocarbonetos aromáticos policíclicos, hidrocarbonetos derivados do petróleo, pesticidas organoclorados, diversos fármacos e outros. Alguns desses compostos são adicionados no processo de fabricação de plásticos, outros são absorvidos ou adsorvidos pelos MPs. ${ }^{12,18,45}$

Segundo Moore, ${ }^{46}$ aproximadamente 267 espécies de organismos marinhos são afetadas por MPs no mundo, porém, esse número pode ser superior ao relatado na literatura, já que ainda não foi avaliada a influência desses materiais em espécies menores. Espécies filtradoras, como os mexilhões, estão propensos a ingerir MPs de tamanho entre 3 e $10 \mu \mathrm{m}$ durante a alimentação; sendo que essas partículas permanecem no marisco por semanas ou meses. ${ }^{47,48}$ von Moos e colaboradores $^{49}$ relataram que MPs podem ser absorvidos pelas brânquias de mexilhões azuis, e após ingeridas se acumulam em vacúolos da glândula digestiva. Quando são ingeridos por organismos de baixo nível trófico, os MPs podem comprometer toda o restante da cadeia alimentar. ${ }^{18}$ Outro exemplo deste efeito devastador dos MPs nos organismos aquáticos é o estudo de Eriksson e Burton, ${ }^{50}$ no qual foi constatado que $97 \%$ do plástico total encontrado nas fezes do lobo-marinho eram partículas de PE e PP. Nesse mesmo panorama, o trabalho de Teuten e colaboradores ${ }^{45}$ revelou a contaminação de um lagarto da índia por PE contendo fenantreno. A Figura 5 mostra as principais fontes que geram MPs (primários e secundários) encontrados nos ambientes aquáticos e a presença destas partículas em alguns organismos vivos relatados na literatura. ${ }^{51}$

Embora o percentual de poluição por MPs proveniente de produtos de cuidados pessoais e cosméticos não seja elevado, não se pode desconsiderar sua ameaça ao ambiente..$^{52} \mathrm{~A}$ grande problemática acerca das microesferas usadas nesses produtos se deve ao fato de poderem ser ingeridas por diversos organismos que em sua maioria está na base das cadeias tróficas. ${ }^{48,49}$ MPs ocasionalmente podem ficar retidos no estômago dos animais, fazendo com que os organismos que ingerem esses animais também sejam contaminados. ${ }^{53}$

É preciso levar em consideração que os plásticos levam em média $4 \%$ de aditivos químicos, como, por exemplo, ftalatos e

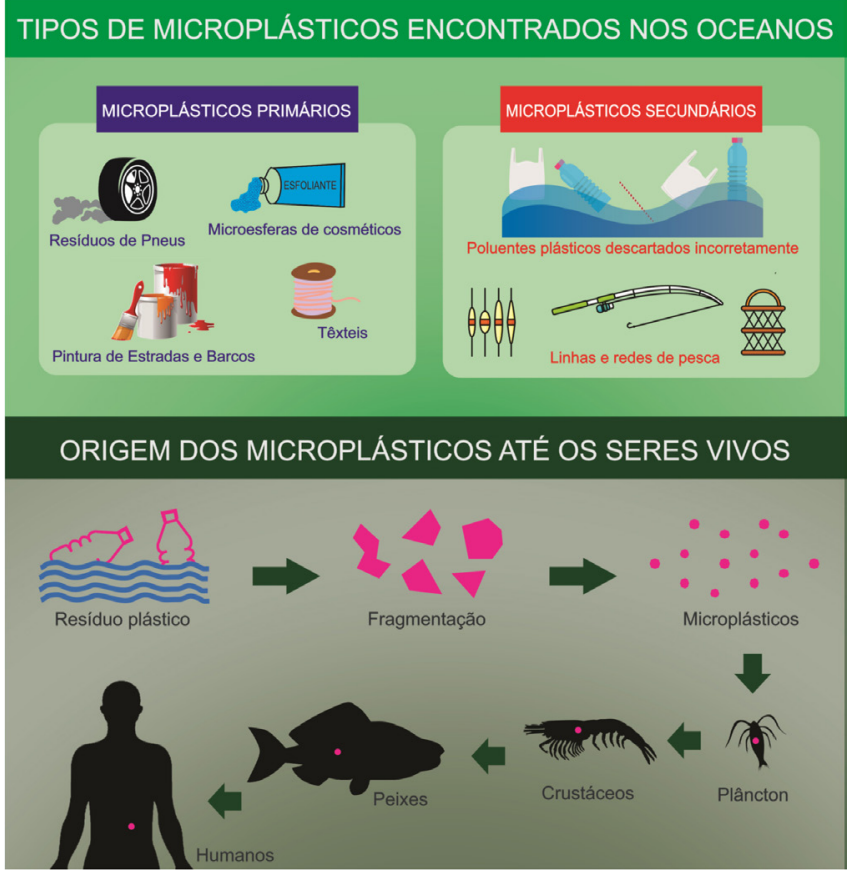

Figura 5. Origem dos MPs (primários e secundários) encontrados nos oceanos, e trajetória até atingir alguns seres vivos

bisfenol. ${ }^{19,54}$ Além disso, a hidrofobicidade dos MPs pode favorecer a adsorção de substâncias tóxicas hidrofóbicas, que são persistentes e bioacomuláveis. ${ }^{18,43,44}$ Ensaios feitos por Engler ${ }^{19}$ demonstraram que tais aditivos químicos e poluentes absorvidos pela superfície de contato do microplástico podem ser lixiviados e transportados para tecidos internos de organismos marinhos que os consomem.

Recentemente, de Faria e colaboradores ${ }^{55}$ avaliaram a presença de MPs nas águas do Pantanal e em um de seus principais afluentes. Os autores encontraram MPs (em forma de fibras e pellets) em afluentes urbanos da cidade de Cuiabá. As principais fontes desses MPs são os esgotos domésticos e estações de tratamento industriais, e provavelmente são transportados até o rio Pantanal. Além disso, MPs derivados de fontes secundárias (resíduos plásticos do turismo e pesca) também podem ser encontrados no Pantanal.

Em suma, faz-se necessário criar legislações que amenizem o impacto ambiental causado por MPs utilizadas em produtos de cuidados pessoais e cosméticos. No Brasil, em 2016, foi proposta uma Lei Federal (PL 6528/2016) que visa proibir produtos de higiene pessoal, cosméticos e perfumaria que sejam fabricados ou manipulados com a adição de microesferas poliméricas. ${ }^{56,57}$ Algumas alternativas para amenizar e substituir as microesferas de plástico seria o uso de diferentes biomateriais, como, por exemplo, cera de abelha, farelo de arroz, amidos derivados de milho, tapioca e carnaúba, algas marinhas, argila e outros compostos naturais.

\section{Interações entre microplásticos e contaminantes: uma abordagem contemporânea}

Em ambientes aquáticos MPs são facilmente transportados devido à elevada persistência, mobilidade e baixa taxa de degradação. Esses materiais são distribuídos e já foram encontrados em estuários, rios, sedimentos, águas profundas dos oceanos e, inclusive, em regiões polares. ${ }^{58,59}$ Isolados MPs são considerados um sério problema ambiental. Porém, quando dispostos em ambientes contaminados com substâncias orgânicas e/ou inorgânicas, o efeito negativo dos MPs é somatizado. MPs atuam como 'acumuladores' e transportadores de diferentes fármacos, metais e outras substâncias tóxicas. ${ }^{60}$ Esses 
materiais com ou sem contaminantes adsorvidos podem ser ingeridos por organismos marinhos, provocando a bioacumulação e propagando efeitos negativos no ecossistema. ${ }^{61,62}$

Entender os possíveis efeitos dessas interações entre microplástico/ contaminantes em sistemas aquáticos se torna essencial e bastante desafiador. Estudos recentes mostram que essas interações dependem de diferentes fatores como $\mathrm{pH}$, temperatura e força iônica do meio, além das propriedades físico-químicas dos envolvidos na interação MPs-poluente..$^{59,61,63,64}$

Assim, esse tópico tem como objetivo apresentar (i) possíveis fatores que podem influenciar o transporte e a interação entre MPs e contaminantes e (ii) mecanismos que favorecem estas interações, como adsorção/dessorção, baseados em estudos e análises de literaturas recentes (estado da arte).

Uma vez lançados no ambiente aquático os MPs podem sofrer modificações em suas propriedades físico-quimicas, devido à alta exposição solar, mudanças de temperatura e movimentações do sistema. ${ }^{62}$ Estudos mostram que essas partículas se tornam mais fragmentadas e com maior quantidade de grupamentos oxigenados quando expostos a radiação ultravioleta. ${ }^{65}$ Além disso, agentes plastificantes como o bisfenol podem ser liberados durante a degradação dos MPs nos ambientes. ${ }^{66}$ Wang e colaboradores ${ }^{65}$ avaliaram os efeitos dos fatores ambientais na adsorção cobre e tetraciclina em micropartículas de PE. Esses pesquisadores verificaram uma maior capacidade adsortiva nos ensaios que foram feitos utilizando as partículas de MPs expostas ao ar, solo e água do que nos ensaios utilizando partículas não modificadas pelos fatores ambientais. Demonstrando que os fatores ambientais alteram a superfície dos MPs, e consequentemente, afetam suas interações com contaminantes.

Partículas de polietileno (PE), poliprolieno (PP), policloreto de vinila (PVC) vêm sendo utilizadas para comprender os mecanismos que envolvem os processos adsortivos de substâncias orgânicos em MPs. A hidrofobicidade e a elevada área superficial dos MPs facilitam a retenção de diferentes poluentes orgânicos, que incluem os hidrocarbonetos aromáticos, fármacos, pesticidas e outros. ${ }^{58,63,64,65}$ Nos ambientes aquáticos, estes compostos orgânicos podem ser complexados pela matéria orgânica dissolvida (MOD) ou adsorvidos nos sedimentos, porém, são facilmente retidos em partículas de MPs devido a elevada afinidade. ${ }^{67} \mathrm{~A}$ Tabela 2 relata a capacidade adsortiva de diferentes contaminantes orgânicos em MPs.

Nos sistemas aquáticos a adsorção/dessorção destes compostos nos MPs é afetada por diferentes fatores, sendo (1) propriedades físico-químicas do MPs: composição, polaridade, área superficial, carga e cristalinidade; (2) condições ambientais: salinidade, temperatura, $\mathrm{pH}$ do meio e presença de MOD e outros poluentes; (3) as propriedades físico-químicas dos poluentes: composição, carga, solubilidade e estabilidade..$^{59,60,67,79}$

Mecanimos de adsorção de contaminantes orgânicos em MPs podem envolver interações hidrofóbicas, atrações/repulsões eletrostáticas, competição pelos sítios de adsorção, dentre outros. Interações hidrofóbicas vêm sendo relatadas como predonimantes na adsorção de orgânicos em MPs. ${ }^{61,62,80}$ Esse mecanimo envolve a partição dos contaminantes entre os MPs e meio aquoso, e está diretamente relacionado ao coeficiente de partição octanol/água $\left(\mathrm{K}_{\mathrm{ow}}\right)$ das substâncias envolvidas. Assim, MPs hidrofóbicos podem adsorver facilmente compostos orgânicos. Porém, é importante ressaltar que a superfície dos MPs é alterada nos ambientes naturais, como, por exemplo, grupamentos oxigenados podem ser adicionados na superfície de MPs modificando suas propriedades e os mecanismos de interação com diferentes substâncias. ${ }^{63}$

As interações também podem ocorrer através da formação de ligações de hidrogênio entre os grupos funcionais dos MPs e
Tabela 2. Capacidades adsortivas de contaminantes orgânicos em MPs

\begin{tabular}{|c|c|c|c|}
\hline MP & $\begin{array}{c}\text { Capacidade adsortiva } \\
\left(\mu \mathrm{g} \mathrm{g}^{-1}\right)\end{array}$ & Poluente orgânico & Ref. \\
\hline PS & 10,80 & triadimenol & 68 \\
\hline PS & 18,94 & miclobutanil & 68 \\
\hline PS & 41,56 & hexaconazol & 68 \\
\hline PS & 8,000 & $17 \beta$-estradiol & 69 \\
\hline PS & 1884 & propanolol & 70 \\
\hline PS & 1882 & sulfametoxazol & 70 \\
\hline PS & 1520 & oxitetraciclina & 71 \\
\hline $\mathrm{PE}$ & 19,50 & $17 \beta$-estradiol & 69 \\
\hline $\mathrm{PE}$ & 64,38 & propanolol & 72 \\
\hline PE & 46,09 & sulfametoxazol & 72 \\
\hline PE & 5852 & ciprofloxacina & 64 \\
\hline $\mathrm{PE}$ & 91,23 & piraclostrobina & 73 \\
\hline PE & 109,0 & tetraciclina & 74 \\
\hline PE & 1670 & tilosina & 75 \\
\hline $\mathrm{PP}$ & 18,00 & $17 \beta$-estradiol & 69 \\
\hline $\mathrm{PP}$ & 113,0 & tetraciclina & 74 \\
\hline $\mathrm{PP}$ & 3330 & tilosina & 75 \\
\hline PP & 402,0 & 3,6 dibromocarbazol & 76 \\
\hline $\mathrm{PP}$ & 415,0 & 1,3,6,8-tetrabromocarbazole & 76 \\
\hline $\mathrm{PVC}$ & 6,000 & $17 \beta$-estradiol & 69 \\
\hline PVC & 202,0 & bisfenol A & 62 \\
\hline PVC & 3330 & tilosina & 75 \\
\hline PVC & 303,0 & fenantreno & 77 \\
\hline $\mathrm{PVC}$ & 350,0 & bifenilpoliclorado & 78 \\
\hline PVC & 150,0 & bisfenol S & 62 \\
\hline PVC & 220,0 & bisfenol B & 62 \\
\hline
\end{tabular}

contaminantes orgânicos. Estudos mostram que esse mecanismo ocorre quando os adsorbato e absorvente possuem grupos doadores/ receptores de prótons como os grupamentos carboxílicos, carbonílicos, aminas ou amidas. ${ }^{62,79,80}$ Quando a superfície do MP é carregada, interações eletrostáticas estão envolvidas no processo adsortivo de substâncias orgânicas em MPs, sendo atrativas (contaminante e MP possuem cargas diferentes) ou repulsivas (contaminante e MP possuem a mesma carga). Forças eletrostáticas podem ser afetadas pelo valor do ponto de carga zero (pcz) do adsorvente e pelo $\mathrm{pH}$ do meio aquoso. ${ }^{61,62,63,79,80}$ Interações $\pi-\pi$ são descritas quando os MPs e substâncias orgânicas possuem em suas estruturas grupamentos aromáticos. ${ }^{81,82}$ A Figura 6 sintetiza os principais mecanismos que controlam o processo adsortivo de contaminantes orgânicos em MPs.

Como mencionado, as condições do meio aquático também afetam a adsorção de contaminantes orgânicos em MPs. Por exemplo, o pH do meio pode alterar a carga de um composto orgânico e afetar a adsorção controlada por interações eletrostáticas. ${ }^{59,82}$ Sais e/ou outros contaminantes presentes no ambiente aquático podem competir com os contaminantes pelos sítios de adsorção. ${ }^{71,83}$ A MOD afeta a adsorção de orgânicos em MPs devido a formação de complexos com os contaminantes ou interação com os MPs. ${ }^{63,84,85}$

Dessa forma, avaliar a adsorção e transporte de contaminantes orgânicos em MPs e seus possíveis efeitos nos ambientes torna-se bastante complicado devido às diferentes condições que controlam esses processos. Muitas vezes, os ensaios em batelada não conseguem reproduzir em detalhes os diferentes fatores que influenciam a 


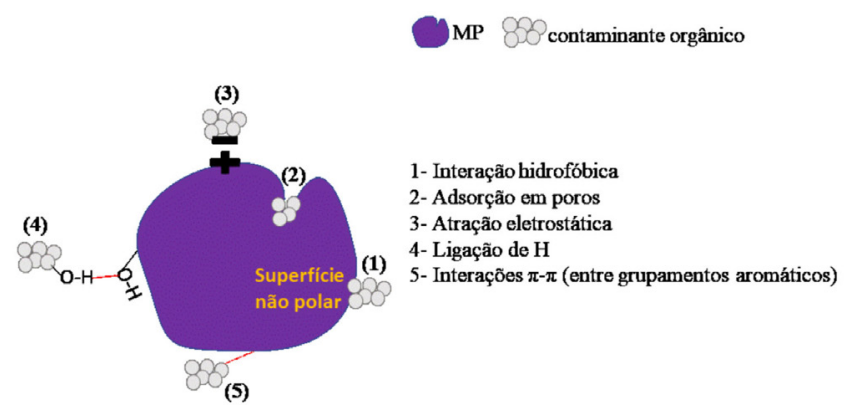

Figura 6. Possíveis mecanismos de adsorção de contaminantes orgânicos em MPs

adsorção, deixando em aberto questionamentos sobre o tópico. Além disso, as caracterizações de amostras com MPs exigem atenção e cautela. Recentemente, Montagner e colaboradores ${ }^{86}$ relataram os desafios envolvendo a quantificação e qualificação de amostras ambientais contendo MPs. No trabalho, os autores reportaram os cuidados a serem tomados durante a amostragem, no preparo das amostras e caracterizações na determinação de MPs em amostras dos diferentes compartimentos ambientais: ar, solo e água. ${ }^{86}$

\section{CONSIDERAÇÕES FINAIS}

A alta produção global de plásticos associado ao manejo inadequado dos resíduos gerados por esses materiais é o principal fator que influencia a difusão/transporte dos microplásticos no ambiente. A bioacumulação dos MPs em organismos marinhos seguida da biomagnificação, representa um risco potencial para a saúde humana.

Salienta-se a carência de pesquisas sobre os impactos ambientais de microfragmentos plásticos em ambientes terrestres e até mesmo no ecossistema aquático de água doce. Em muitas cidades brasileiras, os esgotos domésticos são lançados sem tratamento prévio nos corpos aquáticos, gerando a contaminação de diversos organismos em diferentes níveis tróficos da cadeia alimentar. Um agravante seria falta de saneamento básico, em muitas regiões o tratamento de águas residuais é ineficaz ou inexistente.

Assim como MPs de origem secundária ou primária afetam o ecossistema. Microesferas incorporadas em formulações de produtos de cuidados pessoais e cosméticos constituídas por polietileno e polipropileno merecem atenção e cuidado. Esses materiais podem ser substituídos por esferas de origem natural, como sementes ou cascas mais resistentes. Essa ação diminuiria significantemente o despejo desses fragmentos de plásticos no ambiente.

Ao contrário do monstro relatado na narrativa de Verne, que se apresentava como um objeto maior que uma baleia no fundo do oceano, os "micromonstros" MPs são partículas muito pequenas e muitas vezes invisíveis ao olho humano. Porém, necessitam de muita atenção e novos estudos para que se possa descrever melhor suas interações e transportes de contaminantes tóxicos no sistema aquático. A partir de uma melhor compreensão, novas formas de tratamento poderão ser adotadas nas estações de tratamento visando a eliminação destas partículas da água potável.

\section{AGRADECIMENTOS}

Os autores agradecem o apoio do Conselho Nacional de Desenvolvimento Científico e Tecnológico (CNPq) - n 306271/2017-6, da Fundação de Amparo à Pesquisa do Estado de São Paulo (FAPESP) - n⿳ 2013/20455-2, e da Coordenação de Aperfeiçoamento de Pessoal de Nível Superior - (CAPES) código 001.

\section{REFERÊNCIAS}

1. Verne, J.; Vinte Mil Léguas Submarinas; Vilanova, J. G., trad.; Artes Gráficas Bisordi: São Paulo, 1972.

2. https://iupac.org/, acessado em fevereiro 2022.

3. Vert, M.; Doi, Y.; Hellwich, K. H.; Hess, M.; Hodge, P.; Kubisa, P.; Rinaudo, M.; Schué, F.; Pure Appl. Chem. 2012, 84, 377.

4. Andrady, A. L.; Mar. Pollut. Bull. 2011, 62, 1596.

5. https://www.plasticseurope.org/en, acessada em fevereiro 2022.

6. https://wwf.panda.org/, acessada em fevereiro 2022.

7. Pavoski, G.; Kalikoski, R.; Souza, G.; Baldisserotto, D.; Brum, L. F. W.; Santos, C.; Bergmann, C.; Brandelli, A.; Jacobi, M.; Galland, G. B.; J. Braz. Chem. Soc. 2020, 31, 1566.

8. Lemonick, S.; Chemical and engineering News 2018, 96, 24.

9. Law, K. L.; Thompson, R. C.; Science 2014, 345, 144.

10. Horton, A. A.; Walton, A.; Spurgeon, D. J.; Lahive, E.; Svendsen, C.; Sci. Total Environ. 2017, 586, 127.

11. Hale, R. C.; Seeley, M. E.; La Guardia, M. J.; Mai, L.; Zeng, E. Y.; J. Geophys. Res.: Oceans 2020, 125, 1.

12. Thompson, R. C.; Olsen, Y.; Mitchell, R. P.; Davis, A.; Rowland, S. J.; John, A. W. G.; McGonigle, D.; Russell, A. E.; Science 2004, 304, 838.

13. Wright, S. L.; Thompson, R. C.; Galloway, T. S.; Environ. Pollut. 2013, $178,483$.

14. Li, J. N.; Lusher, A. L.; Rotchell, J. M.; Deudero, S.; Turra, A.; Brate, I. L. N.; Sun, C. J.; Hossain, M. S.; Li, Q. P.; Kolandhasamy, P.; Shi, H. H.; Environ. Pollut. 2019, 244, 522.

15. www.sciencedirect.com, acessada em fevereiro 2022.

16. da Costa, J. P.; Santos, P. S. M.; Duarte, A. C.; Rocha-Santos, T.; Sci. Total Environ. 2016, 566-567, 15.

17. Xu, G.; Cheng, H.; Jones, R.; Feng, Y.; Gong, K.; Li, K.; Fang, X.; Tahir, M. A.; Valev, V. K.; Zhang, L.; Environ. Sci. Technol. 2020, 54, 15594.

18. Fendall, L. S.; Sewell, M. A.; Mar. Pollut. Bull. 2009, 58, 1225.

19. Engler, R. E.; Environ. Sci. Technol. 2012, 46, 12302.

20. Prata, J. C.; Environ. Pollut. 2018, 234, 115.

21. Sleight, V. A.; Bakir, A.; Thompson, R. C.; Henry, T. B.; Mar. Pollut. Bull. 2017, 116, 291.

22. https://cosmeticinnovation.com.br, acessada em fevereiro 2022.

23. https://www.euromonitor.com, acessada em fevereiro 2022.

24. Ribeiro, J. C.; Cosmetologia Aplicada à Dermoestética, 2a ed.; Pharmabooks: São Paulo, 2010.

25. https://www.gov.br/anvisa/pt-br, acessada em fevereiro 2022

26. https://www.institutoecofaxina.org.br, acessada em fevereiro 2022.

27. Gouin, T.; Avalos, J.; Brunning, I.; Brzuka, K.; Graaf, J.; Kaumanns, J.; Konong, T.; Meyberg, M.; Rettinger, K., Schalatter, H.; Thomas, J.; Welie, R.; Wolf, T.; SOFW J. 2015, 3, 40.

28. Eriksen, M.; Mason, S.; Wilson, S.; Box, C.; Zellers, A.; Edwards, W.; Farley, H.; Amato, S.; Mar. Pollut. Bull. 2013, 77, 177.

29. Carr, S. A.; Liu, J.; Tesoro, A. G.; Water Res. 2016, 91, 174.

30. Kalcikova, G.; Alic, B.; Sskalar, T.; Bundschuh, M.; Gotvajn, Z; Chemosphere 2017, 188, 25.

31. Napper, I. E.; Bakir, A.; Rowland, S.; Thompson, R.; Mar. Pollut. Bull. 2015, 99, 178.

32. Bila, D. M.; Pereira, T.; da Silva, A. A.; Resumos do Congresso Brasileiro de Engenharia Sanitária e Ambiental, Natal, Brasil, 2019.

33. Browne, M. A.; Galloway, T.; Thompson R.; Environ. Assess. Manage. 2007, 3, 559 .

34. Chang, M.; Mar. Pollut. Bull. 2015, 101, 330.

35. Duis, K.; Coors, A.; Environ. Sci. Eur. 2016, $28,1$.

36. Zitko, V.; Hanlon, M.; Mar. Pollut. Bull. 1991, 22, 41

37. https://www.gov.uk/government/news/world-leading-microbeads-bancomes-into-force, acessada em fevereiro 2022.

38. Wagner, M.; Scherer, C.; Alvarez-Muñoz, D.; Brennholt, N.; Bourrain, X.; Buchinger, S.; Environ. Sci. Eur. 2014, 26. 
39. Galloway, T.; Thompson, R.; Environ. Sci. Technol. 2011, 45, 9175.

40. Seltenrich, N.; Environ Health Perspect. 2015, 123, A34.

41. http://www.unwater.org/downloads/IYS_flagship_web_small.pdf, acessada em fevereiro 2022.

42. Browne, M.A.; Crump, P.; Niven, S.J.; Teuten, E.; Tonkin, A.; Galloway, T.; Thompson, R.; Environ. Sci. Technol. 2011, 45, 9175.

43. Leslie, H. A.; van der Meulen, M. D.; Kleissen, F. M.; Vethaak, A. D.; Deltares 2011, 104.

44. Rochman, C.M.; Hoh, E.; Hentschel, B.T.; Kaye, S.; Environ. Sci. Technol. 2013, 47, 1646.

45. Teuten, E. L.; Rowland, S. J.; Galloway, T. S.; Thompson, R. C.; Environ. Sci. Technol. 2007, 41, 7759.

46. Moore, C. J.; Environ. Res. 2008, 108, 131.

47. Browne, M. A.; Dissanayake, A.; Galloway, T. S.; Lowe, D. M.; Thompson, R. C.; Environ. Sci. Technol. 2008, 42, 5026.

48. Browne, M. A.; Galloway, T. S; Thompson, R. C.; Environ. Sci. Technol. 2010, 44, 3404.

49. Von Moos, N.; Burkhardt-Holm, P.; Köhler, A.; Environ. Sci. Technol. 2012, 46, 11327.

50. Eriksson, C.; Burton, H.; Ambio 2003, 32, 380.

51. Mercogliano, R.; Avio, C. G.; Regoli, F.; Anastasio, A.; Colavita, G.; Santonicola, S. J.; Agric. Food Chem. 2020, 68, 5296.

52. Guerranti, C.; Martellini, T.; Perra, G.; Scopetani, C.; Cincinelli, A.; Environ. Toxicol. Pharmacol. 2019, 68, 75.

53. EFSA Panel on Contaminants in the Food Chain; Statement on the presence of microplastics and nanoplastics in food, with particular focus on seafood; EFSA J. 2016, 14, 4501, disponível em https://www.efsa. europa.eu/, acessada em fevereiro 2022.

54. Scopetani, C.; Cincinelli, A.; Martellini, T.; Lombardini,E.; Ciofini, A.;Fortunati, A.; Pasquali, V.; Ciattini, S.; Ugolini, A.; Environ. Res. 2018, $167,411$.

55. de Faria, E.; Girard, P.; Nardes, C. S.; Moreschi, A.; Christo, E. S; Junior, L. F.; Costa, M. F.; Environ. Engin. 2021, 3, 100088.

56. https://www.camara.leg.br/proposicoesWeb/fichadetramitacao?idProp osicao=2117806, Agência Câmara de Notícias, acessada em fevereiro 2021.

57. https://www.camara.leg.br/noticias/561049-projeto-que-proibemicroesferas-de-plastico-em-cosmeticos-e-aprovado-na-2a-comissao/, Agência Câmara de Notícias, acessada em fevereiro 2022.

58. Qiua, Y; Zhengb, M.; Wanga, L.; Zhaoa, Q.; Loua, Y.; Shia, L.; Qu, L.; Mar. Pollut. Bull. 2019, 146, 718.

59. Vieira, Y.; Lima, E. C.; Foletto, E. L.; Dotto, G. L.; Sci. Total Environ. 2021, 753, 141981.

60. Guo, X.; Wang, J.; Mar. Pollut. Bull. 2019, 142, 1-14.

61. Liu, F.; Liu, G.; Zhu, Z.; Wang, S.; Zhao, F.; Chemosphere 2019, 214, 688.
62. Wu, P.; Cai, Z.; Jin, W.; Tang, Y.; Sci. Total Environ. 2019, 650, 671.

63. Li, J.; Zhang, K.; Zhang, H.; Environ. Pollut. 2018, 237, 460.

64. Atugoda, T.; Wijesekara, H.; Werellagama, D. R. I. B.; Jinadasa, K. B. S. N.; Bolan, N. S.; Vithanage, M.; Environ. Technol. Innovation 2020 , 19, 100971.

65. Wang, Y.; Wang, X.; Li, Y.; Li, J.; Liu, Y.; Xi; S.; Zhao, J.; Chem. Eng. J. 2021, 404, 126412.

66. Yu, Z.; Peldszus, S.; Huck, P. M.; Water Res. 2008, 42, 2882.

67. Joo, S.H.; Liang, Y.; Kim, M.; Byun, J.; Choi, H.; Environmental Challenges 2021, 3, 100042.

68. Fang, S.; Yu, W.; Li, C.; Liu, Y.; Qiu, J.; Kong, F.; Sci. Total Environ. 2019, 691, 119.

69. Liu, X.; Xu, J.; Zhao, Y.; Shi, H.; Huang, C.; Chemosphere 2019, 226, $726 \mathrm{e} 735$.

70. Huang, Y.; Ding, J.; Zhang, G.; Liu, S.; Zou, H.; Wang, Z.; Zhu, W.; Geng, J.; Sci. Total Environ. 2021, 752, 142256.

71. Zhang, H.; Wang, J.; Zhou, B.; Zhou, Y.; Dai, Z.; Zhou, Q.; Chriestie, P.; Luo, Y.; Environ. Pollut. 2018, 243, 1550.

72. Razanajatovo, R. M.; Ding, J.; Zhang, S.; Jiang, H.; Zou, H.; Mar. Pollut. Bull. 2018, 136, 516.

73. Hai, N.; Liu, X.; Li, Y.; Kong, F.; Zhang, Y.; Fang, S.; ACS Omega 2020, 5,30679 .

74. Xu, B.; Liu, F.; Brookes, P.C.; Xu, J.; Environ. Pollut. 2018, 240, 87.

75. Guo, X.; Pang, J.; Chen, S.; Jia, H.; Chemosphere 2018, 209, 240.

76. Zhang, X.; Zheng, M.; Yin, X.; Wang, L.; Lou, Y.; Qu, L.; Liu, X.; Zhu, H.; Qiu, Y.; Mar. Pollut. Bull. 2019, 138, 458.

77. Wang, W.; Wang, J.; Ecotoxicol. Environ. Saf. 2018, 147, 648.

78. Zhan, Z.; Wang, J.; Peng, J.; Xie, Q.; Huang, Y.; Gao, Y.; Mar. Pollut. Bull. 2016, 110, 559.

79. Wang, F.; Zhang, M.; Sha, W.; Wang, Y.; Hao, H.; Dou, Y.; Li, Y.; Molecules 2020, 25, 1827.

80. Guo, X.; Wang, J.; Mar. Pollut. Bull. 2019, 142, 1.

81. Liu, G.; Zhu, Z.; Yang, Y.; Sun, Y.; Yu, F.;Ma, J.; Environ. Pollut. 2019, 246, 26.

82. Velzeboer, I.; Kwadijk, C.J.; Koelmans, A. A.; Environ. Sci. Technol. 2014, 48, 4869.

83. Bakir, A.; Rowland, S. J.; Thompson, R. C.; Estuarine, Coastal Shelf Sci. 2014, 140, 14.

84. Xu, B.; Liu, F.; Brookes, P. C.; Xu, J.; Environ. Pollut. 2018, $240,87$.

85. Zhang, H.; Wang, J.; Zhou, B.; Zhou, Y.; Dai, Z.; Zhou, Q.; Chriestie, P.; Luo, Y.; Environ. Pollut. 2018, 243, 1550.

86. Montagner, C. C.; Dias, M. A.; Paiva, E. M.; Vidal, C.; Quim. Nova 2021, 44, 1328 